\section{Bioinspired wing and tail morphing extends drone flight capabilities}

\author{
Enrico Ajanic $^{1 *}$, Mir Feroskhan ${ }^{2}$, Stefano Mintchev ${ }^{3,4}$, Flavio Noca ${ }^{5}$, Dario Floreano ${ }^{1 *}$
}

\begin{abstract}
The aerodynamic designs of winged drones are optimized for specific flight regimes. Large lifting surfaces provide maneuverability and agility but result in larger power consumption, and thus lower range, when flying fast compared with small lifting surfaces. Birds like the northern goshawk meet these opposing aerodynamic requirements of aggressive flight in dense forests and fast cruising in the open terrain by adapting wing and tail areas. Here, we show that this morphing strategy and the synergy of the two morphing surfaces can notably improve the agility, maneuverability, stability, flight speed range, and required power of a drone in different flight regimes by means of an avian-inspired drone. We characterize the drone's flight capabilities for different morphing configurations in wind tunnel tests, optimization studies, and outdoor flight tests. These results shed light on the avian use of wings and tails and offer an alternative design principle for drones with adaptive flight capabilities.
\end{abstract}

\section{INTRODUCTION}

Fixed-wing drones play an increasing role in civilian applications, such as disaster mitigation, environmental monitoring, inspection, and delivery (1). Their aerodynamic efficiency enables fast cruise flight for covering larger distances with lower energy expenditure than multicopters of the same mass. However, fixed-wing drones still struggle when navigating in complex, obstacle-rich environments, such as cities $(2,3)$.

For aggressive flight in complex environments, fixed-wing drones must perform sudden and sharp course variations at a wide range of flight speeds. Such flight capabilities require high maneuverability and agility. Maneuverability is defined as the ability to rapidly change the linear velocities acting on the airframe (Materials and Methods) $(4,5)$. The change in linear velocity is greatest when a strong propulsive force is applied or the aerodynamic forces, such as lift and drag produced by the lifting surfaces, are large. Agility is defined as the ability of rapidly changing the rotational rate acting on the airframe (Materials and Methods) $(5,6)$. The change in rotational rate is greatest when the turning moments produced by the lifting surfaces (wing and tail) are large, the airframe's moment of inertia is small, and the inherent stability is low $(7,8)$. Instead, during cruise, flight drones operate mostly in steady-state flight; they must fly fast and at a low cost of energy. A high inherent stability is favored because it helps to maintain equilibrium flight conditions and to decrease the sensitivity to wind gusts (9). Furthermore, the required power for flight should be optimized by improving the lift-to-drag ratio $(10,11)$. A fixed-wing drone cannot effectively resolve these aerodynamic requirements between aggressive and cruise flight because the fixed geometry of its lifting surfaces excels only within a small range of operating conditions $(12,13)$.

\footnotetext{
${ }^{1}$ School of Engineering, Swiss Federal Institute of Technology Lausanne, Lausanne, Switzerland. ${ }^{2}$ School of Mechanical and Aerospace Engineering, Nanyang Technological University, Singapore, Singapore. ${ }^{3}$ Department of Environmental Systems Science, Swiss Federal Institute of Technology Zurich, Zurich, Switzerland. ${ }^{4}$ Swiss Federal Institute for Forest, Snow and Landscape Research, Birmensdorf, Switzerland. ${ }^{5} \mathrm{HES}-\mathrm{SO}$ University of Applied Sciences and Arts Western Switzerland, Geneva, Switzerland.

*Corresponding author. Email: enrico.ajanic@epfl.ch (E.A.); dario.floreano@epfl.ch (D.F.)
}

Research suggests that birds deal with this challenge by morphing their wings and tails, which greatly benefits their flight performance when gliding $(14,15)$. Birds sweep the main wing forward and fan the tail outward to produce considerable lift and drag during slow and aggressive flight to increase maneuverability (Fig. 1A) (16). Here, research suggests that the tail supports the main wing in generating additional lift and drag, analogous to trailing edge flaps of aircraft wings $(17,18)$. To increase agility, birds enlarge and deflect their wings and tails to produce considerable aerodynamic moments (Fig. 1A) $(19,20)$. The relatively short bodies of birds, which resemble more flying wings than traditional aircrafts, and their lightweight wings contribute to lower inherent inertia, which further enhances agility (21-23). Furthermore, in aggressive flight, birds actively reduce longitudinal stability by sweeping the main wings forward and minimizing the tail's surface $(24,25)$. However, in fast cruise flight (Fig. 1A), birds decrease the size of wing and tail surfaces and sweep the main wing backward to generate a streamlined profile, thus minimizing the required power for flight (26-28). This backwardswept wing configuration also increases the inherent longitudinal stability (14).

The aerodynamic adaptability of birds has inspired researchers to apply avian-inspired morphing strategies to drones $(10,29-39)$. Previous work has studied the effects of area change on maneuverability (10), asymmetric sweep on roll control $(10,34)$, and forward sweep on pitch control $(31,32)$. However, the combined application of a wing capable of changing its area and of sweeping forward, as seen on birds, has not been studied for drones. Furthermore, all previous experimental studies, including our own (10), have been limited to the main wing. The interplay of a morphing wing with a bird-like, morphing tail and its potential to increase flight performance has not yet been addressed in aerial robotics.

Here, we experimentally investigate the aerodynamic effects of avian-inspired morphing of wing and tail (Fig. 1A) and show that it can increase drone flight capabilities by adapting the aerodynamic profile to contrasting flight conditions, namely, aggressive and cruise flight (Fig. 1B). We develop a drone whose wing and tail dimensions approximate those of the northern goshawk (Accipiter gentilis), a bird of prey with a relatively long tail and long wing chord, which permit great morphological adaptions (Fig. 1A) (40). As a result, the bird is capable of both quick maneuvering when hunting in highly 

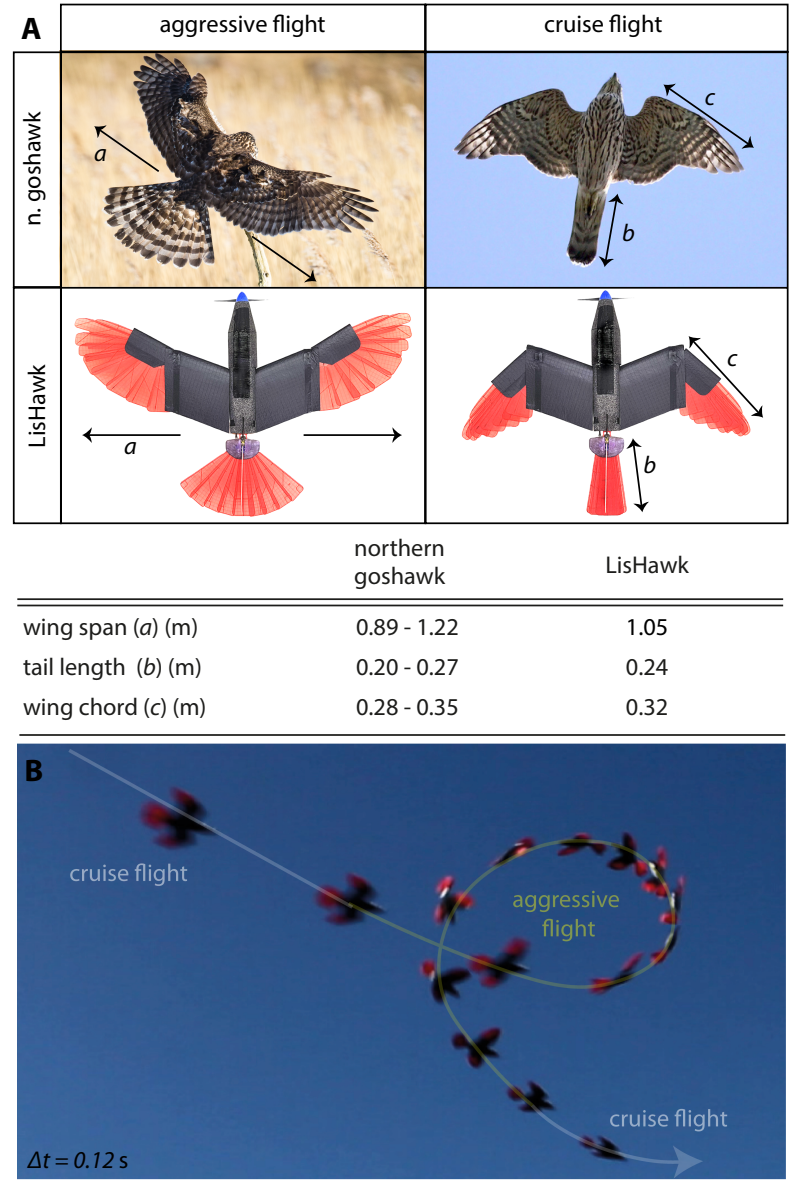

Fig. 1. Avian-inspired wing and tail morphing. (A) Like the northern goshawk, the LisHawk's lifting surfaces consist of a relatively long tail and short wings with a large wing chord that enable large geometrical changes to efficiently adapt between aggressive and cruise flight $(41,48,57)$. Photo credits: $(58,59)$. (B) LisHawk drone changing between cruise and aggressive flight during flight tests.

cluttered environments, such as forests, and fast gliding flight when silently approaching prey in the open terrain $(41,42)$. By example of the LisHawk drone, we apply this avian-inspired morphing strategy and show improved agility, maneuverability, inherent stability, flight speed range, and power requirement when actively changing the shape of the wing and tail. The experimental method consists of the aerodynamic characterization, morphology optimization, and flight tests with different morphing configurations.

\section{RESULTS}

\section{Avian-inspired drone}

To characterize the aerodynamic effects of avian-inspired wing and tail morphing and to test the design principles on a robotic system, we developed a drone, code-named LisHawk (Fig. 1A), with a ready-to-fly weight of $284 \mathrm{~g}$ and a maximum wingspan of $1.05 \mathrm{~m}$. Besides the adoption of the avian morphing strategy to improve the aerodynamic performance, we were also inspired by the avian body structure. The northern goshawk's body is composed of rigid, lightweight bones that absorb loads and aerodynamic surfaces made of soft flesh and feathers (43), which has a low mass and moment of inertia (23). Similarly, we designed a skeleton out of fiber-reinforced plastics, which offers high strength at a low weight. A durable, flexible, and lightweight expanded polypropylene (EPP) body encapsulates this skeleton and protects it from impact (Fig. 2A). We also placed the avionics ( $52 \%$ of the overall drone weight) close to the drone's center of gravity (Fig. 2A) to further reduce the moment of inertia. Unlike the goshawk, which generates thrust by flapping its wings, we used a tractor propulsion system consisting of an electrical motor and a propeller. This design choice allowed us to focus the experimental characterization of our avian morphing strategy on the control of fewer degrees of freedom, while obtaining higher propulsive efficiency at comparatively lower system complexity $(44,45)$.

Furthermore, the morphing surfaces are made from discrete, feather-like plates (Fig. 2B) that we used in previous work (10). These artificial feathers can be designed in a large variety of dimensions and shapes, making them suitable for wing and tail; their properties are replicable because of precise manufacturing; and their partially soft architecture offers durability. In comparison, biological feathers offer comparatively lower weight, higher softness, and self-healing properties (34). However, their shape variations both within each bird and across birds of the same species cause a lengthier manufacturing process, without mentioning ethical issues involved in adopting the proposed design for large-scale production of feathered drones.

\section{Morphing wing and tail architecture}

During gliding flight, birds such as the northern goshawk mainly rely on the folding of their wrists to change the wing area (40). Similarly, we equipped the LisHawk's wing with folding outer sections, thus affecting sweep angle and surface area (Fig. 2C and movie S3). The morphing region of each wing side consists of nine artificial feathers (I to IX), which fan outward when the wing is extended and overlap each other when the wing is tucked (Fig. 2G). The outermost feather (I) is fixed to the skeleton, while the inner feathers (II to IX) can rotate in the wing plane around their feather pins. To actuate feathers II to IX, we connected the feather shafts to an elastic tendon (Fig. $2 \mathrm{H}$ ), which guarantees a regular feather spacing in the wing plane when the wing is extended (10). The elastic connector is prestretched (preload, $0.65 \mathrm{~N}$ ) to overcome the surface friction of the overlapping feathers when the wing is tucked. This avian-inspired, underactuated design is lightweight, and the softness of the morphing surfaces offers mechanical robustness (34). Each side of the wing is independently actuated by a separate servomotor through the wing servo linkage (Fig. 2G). When extending the wing, this servomotor must overcome the elastic tendon's spring force $(2.35 \mathrm{~N}$ when extended) and the aerodynamic forces. Hence, an active load must be applied to extend the wing, whereas only a small load is required when the wing is tucked. The synchronous activation of the two wing sides generates a symmetric sweep variation, resulting in a maximum wing area change of $41 \%$ (Fig. 1C), a shift in center of gravity (Fig. 2D, blue line), and a change in moment of inertia (Fig. 2D, red lines). Asynchronous activation generates asymmetric sweeping between the two wing sides, resulting in a maximum area difference between left and right wing of $41 \%$, a shift in center of gravity (Fig. 2E, blue line), and a change in moment of inertia (Fig. 2E, red lines). The wing area divergence produces a moment around the aircraft's $x$ axis in flight, which is used to control the roll angle, as previously shown in $(10,34)$.

The LisHawk's feathered tail can fold and act as an elevator (Fig. 2F and movie S3). Instead of adding twisting capabilities as seen 
A

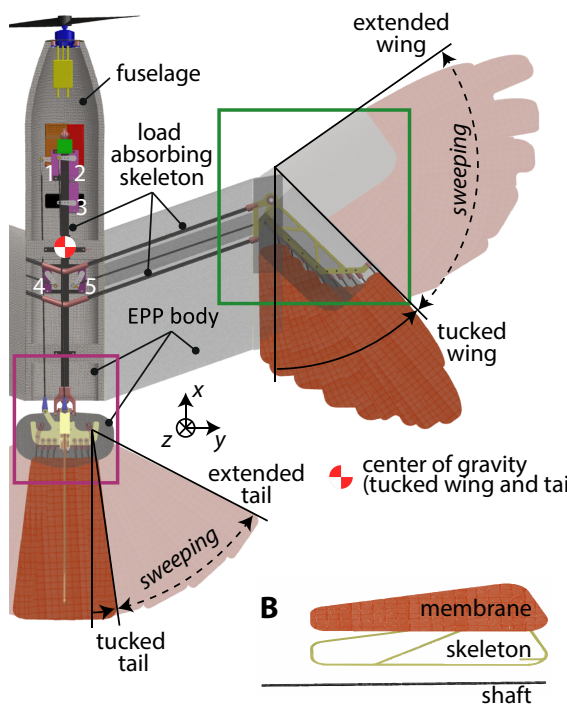

C

\begin{tabular}{lcc} 
property & \multicolumn{2}{c}{ measurement } \\
& tucked & extended \\
\hline \hline wing angle $\left(^{\circ}\right)$ & 45 & 130 \\
wing area $\left(\mathrm{m}^{2}\right)$ & 0.117 & 0.165 \\
tail angle $\left(^{\circ}\right)$ & 10 & 60 \\
tail area $\left(\mathrm{m}^{2}\right)$ & 0.014 & 0.044 \\
wing \& tail area $\left(\mathrm{m}^{2}\right)$ & 0.131 & 0.209 \\
\hline
\end{tabular}

D

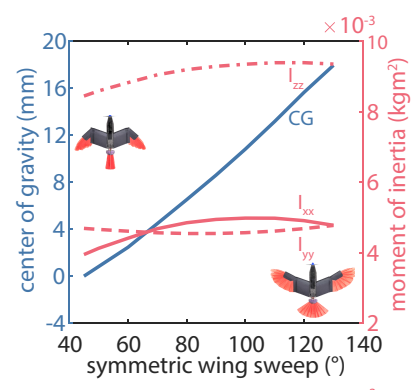

$\mathbf{E}$

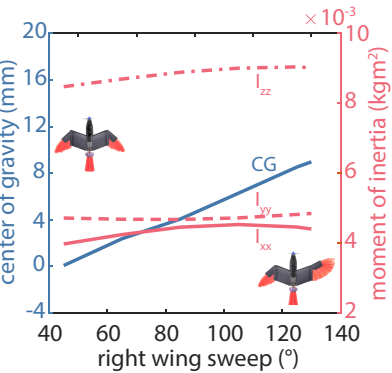

$\mathbf{F}$

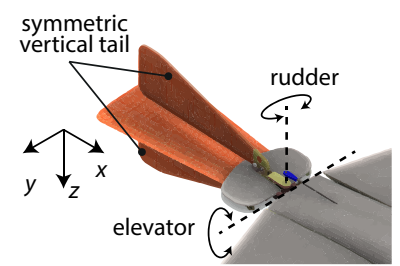

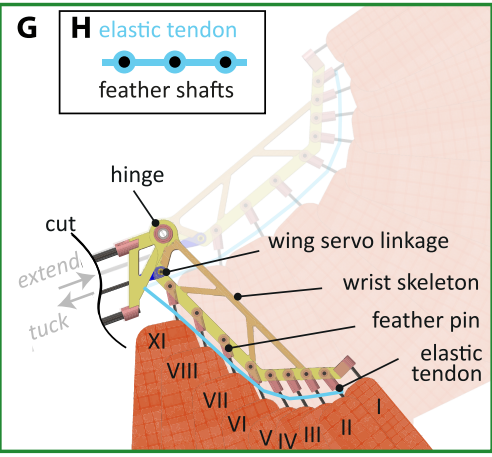

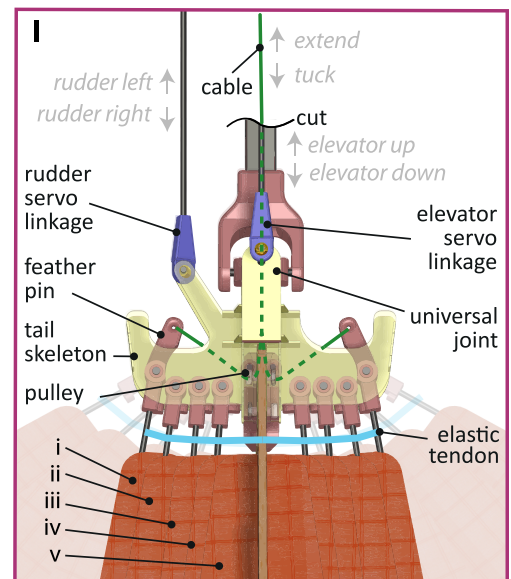

Fig. 2. LisHawk morphing platform architecture. (A) Top view of the LisHawk drone with a partially transparent body to show the load-absorbing skeleton, the location of the avionics (see Materials and Methods), and the morphing capabilities of wing and tail (movie S3). The top two servomotors (purple) actuate the rudder (1) and the elevator (2), the middle servomotor actuates the tail spread (3), and the bottom two servomotors actuate the left (4) and right (5) wing sweeping (see table S1 for fullstroke actuation times). (B) The artificial feathers consist of three parts: a durable ripstop membrane, a flexible glass fiber skeleton, and a stiff carbon shaft. (C) Key geometrical parameters of the morphing wing and tail. (D) Shift in center of gravity (CG; blue line) and moment of inertia (red lines) caused by symmetric wing morphing [computer aided design (CAD) data]. (E) Shift in center of gravity (CG; blue line) and moment of inertia (red lines) caused by asymmetric wing morphing (CAD data). The left wing was tucked, while the right wing was morphed from tucked to extended. (F) The morphing tail can deflect in the $x z$ plane to act as an elevator and in the $x y$ plane to act as a rudder by deflecting the vertical tail (movie S3). (G) Top view of the right-wing morphing mechanism (see Materials and Methods and movie S1). For the sake of clarity, the EPP body is removed. (H) A 3D-printed elastic tendon connects the feather shafts and distributes the feathers equally in the wing plane. (I) Top view of the tail morphing mechanism (see Materials and Methods for further information). For the sake of clarity, the body is removed.

on birds (19), which would have been mechanically complex, we let the tail induce a yaw moment by adding a vertical fin (Fig. 2F) that can be deflected in the $x y$ plane (Fig. 2I and movie S3). The elevator and rudder deflections are facilitated by a universal joint and are actuated by servomotors through carbon linkages. Our morphing tail consists of nine artificial feathers: one fixed central feather (v) and four feathers on each side of the central feather (i to iv), which can rotate around the feather pins in the tail plane (Fig. 2I). Both sides of the tail are actuated in symmetry. Analogous to the morphing wing, we interconnected the tail feathers with a prestretched (preload, $0.92 \mathrm{~N}$ ) elastic connector for even spacing in the tail plane and enabled the two outermost feathers (i) to guide the inner feathers (ii to iv) when extending the tail (Fig. 2I). The outer feathers (i) are actuated via a cable that is guided over pulleys to a servomotor (Fig. 2A). As for the morphing wing, a load must be applied when extending the tail to stretch the elastic tendon $(3.43 \mathrm{~N}$ when extended) and to overcome aerodynamic forces, whereas only a small load is required when the tail is tucked. The morphing tail achieves an area change of $214 \%$ when changing from tucked to extended.

\section{Wing and tail morphing increases maneuverability}

Maneuverability describes the drone's control ability to change the velocity vector (4), which is greatest when the linear accelerations acting on the airframe are maximized. Wing and tail morphing as applied on the LisHawk alters the lift and drag by changing the shape and area of the aerodynamic surfaces (Fig. 3B). To understand the direct implication of these forces on maneuverability, we considered the aircraft equations of motion in the flight path direction with zero sideslip condition and no thrust (see Materials and Methods for the equation and Supplementary Text for its derivation). We can show that the only external factors affecting the linear accelerations are the resultant lift and drag forces, which should be large. Therefore, we placed the LisHawk drone in the wind tunnel and measured the lift and drag forces resulting from symmetric wing and tail morphing (Fig. 3A), and tail elevation (Fig. 3D).

Our experiments show that changing the wing and tail from tucked to extended configuration greatly increases lift and drag (Fig. 3C, black to red line) due to the increased wing and tail area. As commonly observed in the low Reynolds number regime $\left(<10^{5}\right)$, the maximum lift is reached at high angles of attack $\left(24^{\circ}\right.$ and above) 
A
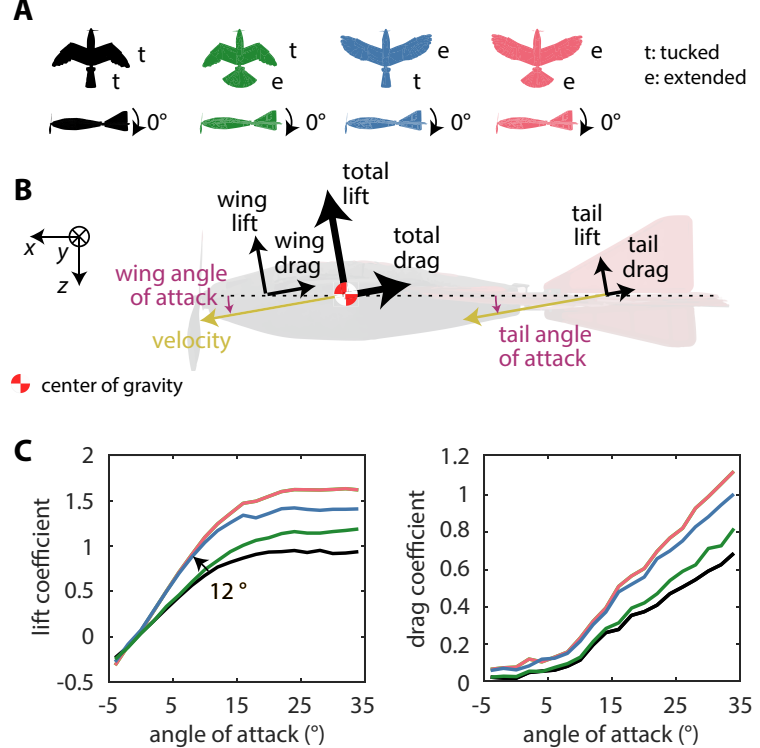

D
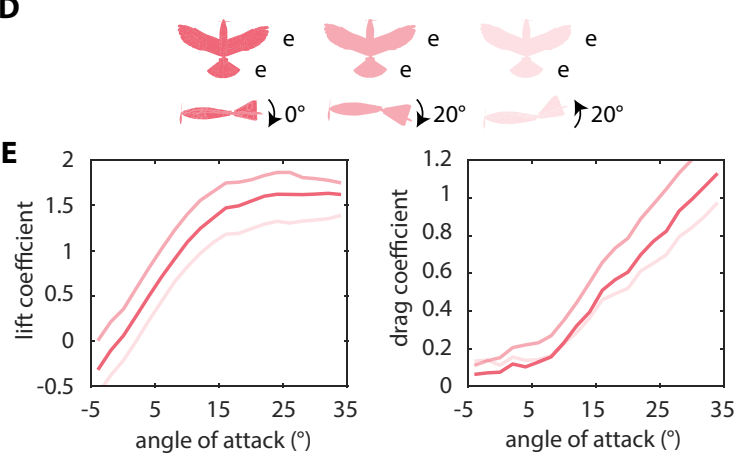

Fig. 3. Wing and tail morphing improves maneuverability. Static wind tunnel data (flow velocity of $8.0 \mathrm{~m} / \mathrm{s}$ ). (A) In the first experiment, we applied four different wing and tail configurations: tucked wing and tail (black), tucked wing and extended tail (green), extended wing and tucked tail (blue), and extended wing and extended tail (red), while the tail has a zero deflection (wing and tail angle of attack are the same). (B) Free-body force illustration of the wing's (including fuselage) and tail's lift and drag components, which are superimposed to obtain the total lift and drag force. We presume that the drag force is parallel to the velocity vector such that the velocity vector deviates from the $x$ axis by the angle of attack in the $x z$ plane, while lift is perpendicular to the velocity vector and the $y$ axis. (C) Extending wing and tail increases the lift (left) and drag (right) for all positive measured angles of attack. (D) In the second experiment, we applied zero elevation (red), $20^{\circ}$ downward elevation (light red), and $20^{\circ}$ upward elevation (very light red), while the wing and tail were fully extended. (E) Deflecting the tail downward increases the lift (left) and drag (right) over the measured angle of attack range (extended wing and tail configuration).

for all configurations and remains nearly constant beyond that angle, while drag further increases (46). When measuring the wing's contribution to the aerodynamic forces (Fig. 3B), we find that extending the wing steepens the lift slope by $12^{\circ}$, thus increasing lift with respect to the angle of attack, while drag is increased over the entire range of angle of attack (Fig. 3C, blue line). Enlarging the tail increases lift and drag only above $14^{\circ}$ (Fig. 3, B and C, green line). We think that this may be due to disturbed flow conditions behind the fuselage's and wing's wake, which affects the adjacent morphing tail (22).

Moreover, deflecting the tail downward, and thus increasing the tail's angle of attack, notably contributes to positive lift and drag
(Fig. 3E, light red line) over the entire angle of attack range. Deflecting the tail upward, which decreases the tail's angle of attack, also decreases the lift, while the drag is increased at low angles of attack (up to $8^{\circ}$ ) and increased beyond that angle (Fig. 3E, lightest red line). For all other tested wing and tail morphologies (Fig. 3A), a similar trend is observed, while a smaller tail also produces a comparatively smaller change in lift and drag force (fig. S4).

\section{Wing and tail morphing increases agility}

Next, we examined the increase in agility, which is the drone's control ability to change the angular rate, because of wing and tail morphing. Agility is greatest when the angular accelerations acting on the airframe are maximized. When considering a pure pitching, rolling, or yawing motion, angular acceleration is defined as the aerodynamic moment (pitch, roll, or yaw) divided by the moment of inertia about the corresponding axis of motion (Eq. 6, Materials and Methods). Therefore, we can deduce that the angular acceleration is greatest when the aerodynamic moments are large and the moments of inertia are small. To estimate the impact of avianinspired wing and tail morphing on agility, we placed the LisHawk drone in the wind tunnel and measured the moments resulting from symmetric wing morphing, tail morphing, and tail elevation (Fig. 4A), as well as asymmetric wing morphing (Fig. $4 \mathrm{G}$ ). In this section, we will focus on pitch-up motion (Fig. 4, A to F) and roll motion (Fig. 4, G to I), which are key factors when performing agile maneuvers such as perching or rapid turning (19).

Let us first consider the effects of morphing aerial surfaces on the pitch-up moment. In fixed-wing aircraft, a pitch-up moment is typically produced by deflecting the tail upward, which results in a negative lift force away from the center of gravity (tail moment arm) (Fig. 4B). The LisHawk drone can further increase this pitch-up moment by extending its wing and tail: Extending the tail increases the negative aerodynamic forces, whereas extending the wing increases the wing lift force and the wing moment arm (Fig. 4B), which we could confirm through our wind tunnel experiments. In the condition where the tail is extended, the measured pitch coefficient is notably larger and remains positive for a larger range of angles of attack (Fig. 4C, green line), as compared with the tucked wing and tail configuration (Fig. 4C, black line). In the condition where the wing is extended, the measured pitch coefficient is large (although smaller than with the tucked wing and extended tail below $12^{\circ}$ ), and importantly, it remains large even at $34^{\circ}$ incidence (Fig. 4D, blue line). In the condition where both wing and tail are extended, the measured pitch-up coefficient is the largest for intermediate angles of attack and remains positive across the entire range of angles of attack (Fig. 4E, red line). It is therefore possible to adapt the shape of wing and tail to the drone's angle of attack to produce maximum pitch-up moments (Fig. 4F).

Moreover, it is important to note that pitch agility can increase maneuverability: A rapid change in pitch speed temporarily increases the angle of attack. The increased angle of attack leads to an increased lift force and can be further reinforced by increasing the wing and tail area from tucked to extended (Fig. 3C).

In fixed-wing aircraft, roll moments are typically generated by ailerons. However, ailerons are prone to large roll moment reduction or even reversal at high angles of attack (47). Instead, in birds and in our avian-inspired drone, roll motion can be produced by asymmetric sweeping of the main wing. We measured the roll coefficient by placing the drone in the wind tunnel (Materials and Methods) 

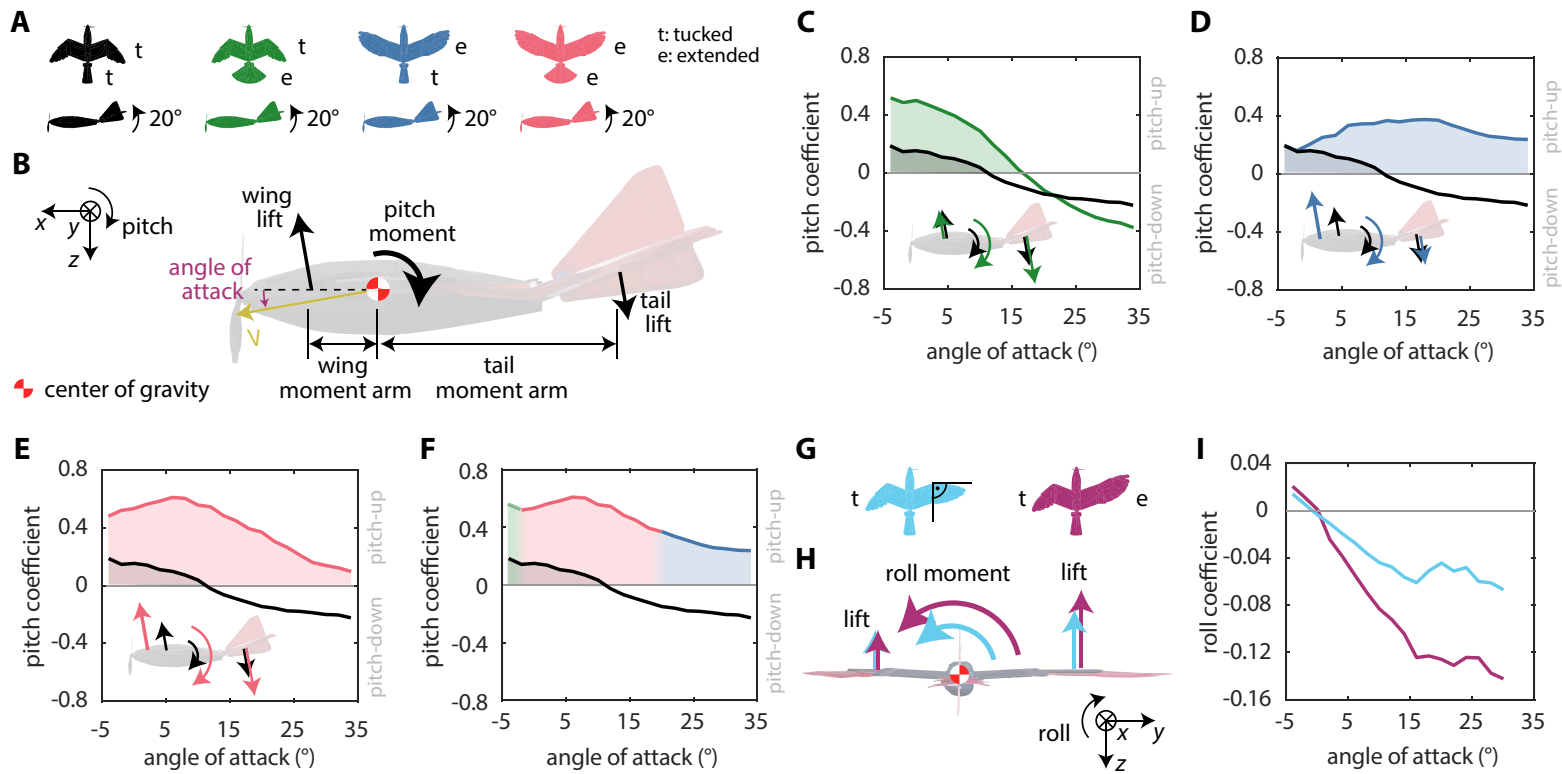

Fig. 4. Wing and tail morphing improves agility. Static wind tunnel data (flow velocity of $8.0 \mathrm{~m} / \mathrm{s}$ ). The shaded area indicates the pitch-up moment, and gray lines indicate the zero moment. The pitch coefficient curves of the downward deflection can be found in fig. S5. (A) In the first experimental setup, we applied four different wing and tail configurations: tucked wing and tail (black), tucked wing and extended tail (green), extended wing and tucked tail (blue), and extended wing and extended tail (red), while the tail has a $20^{\circ}$ upward deflection. (B) The lift forces of wing (including fuselage) and tail act away from the center of gravity by a wing and tail moment arm, respectively, causing a pitching moment around the body's $y$ axis. (C) The relatively low positive pitch coefficient when wing and tail are tucked (black line) is notably increased when extending the tail (green line). (D) The drone's limitation of producing a positive pitch moment only at low angles of attack when wing and tail are tucked (black line) is overcome by extending the wing (blue line). (E) Extending wing and tail (red line) notably increases the pitch coefficient over the entire angle of attack range. (F) The synergy between morphing wing and tail permits large pitch-up moments over a greater angle of attack range. (G) To quantify the roll moment, we apply two different wing asymmetries: left wing tucked and right wing at $90^{\circ}$ (teal) and left wing tucked and right wing extended (purple) (movie S3). (H) The tucked left side of the wing produces a smaller lift force than the right extended wing, which induces a roll motion to the left. (I) Both wing asymmetries show a high roll coefficient at high angles of attack.

for two asymmetric sweep configurations of the main wing (Fig. 4G). For a counterclockwise roll motion, the negative roll moment generated by the asymmetric sweep increases at higher angles of attack and is larger for higher sweep asymmetry (Fig. 4I). These results suggest that asymmetric sweeping can produce higher roll agility in aggressive flight when the angles of attack are high, which could also be verified during flight tests (movie S5).

\section{Wing and tail morphing affects pitch stability}

Pitch stability describes the aircraft's inherent tendency to return to trim (equilibrium flight condition; Fig. 5A, top) after an external disturbance such as a wind gust. The tendency to return to trim flight results from the aft location of the total aerodynamic force with respect to the center of gravity, which produces a restoring pitch moment (Fig. 5A, middle). This phenomenon is desirable in cruise flight because it does not require active control to return to trim flight (8). In contrast, pitch instability results from the frontal location of the total aerodynamic force with respect to the center of gravity. Pitch instability is desirable in aggressive flight because of its tendency to magnify control inputs but often requires a sophisticated control system for stabilization (Fig. 5A, bottom) (48).

In contrast to agility, where we considered the magnitude of the pitch coefficient because of a control input (tail elevation and wing sweeping), when analyzing pitch stability, we consider the slope of the pitch coefficient (Fig. 5, C to E) at increasing angles of attack (see Materials and Methods for mathematical formulation). A negative pitch coefficient slope corresponds to an increasing restoring moment and pitch stability (Eq. 7, Materials and Methods).
In the control condition, where both wing and tail are tucked (Fig. 5B), the trim angle is $2^{\circ}$ (Fig. 5C, black circle) and the pitch coefficient slope is negative (stable) for increasing angles of attack (Fig. 5C, black line). In the condition where the tail is extended, the trim angle remains almost the same, but the pitch coefficient slope is more negative (Fig. 5C, green line) because of the tail's increased lift and drag. This decreased pitch coefficient slope results in a higher restoring moment, which increases the drone's tendency of returning to equilibrium after a disturbance. Instead, in the extended wing and tucked tail condition, the pitch coefficient slope is positive (unstable) up to an angle of attack of $20^{\circ}$ (Fig. 5D, blue line), which is caused by the forward shift of the total aerodynamic force with respect to the center of gravity (Fig. 5A, bottom) (see Supplementary Text). Beyond $20^{\circ}$, however, the pitch coefficient slope becomes negative and the drone is stable. Last, in the condition where both tail and wing are extended, the pitch stiffness is positive up to an $8^{\circ}$ angle of attack before transitioning into negative at increasing angles of attack (Fig. 5E, red line). The trim point is shifted to $20^{\circ}$ (Fig. 5E, red circle). These results indicate that avianinspired wing and tail morphing can notably change the drone pitch stability depending on cruise or aggressive flight, and even shift trim to high angles of attack, where the high lift and drag allow very slow flight.

\section{Wing and tail morphing reduces power requirement and increases speed range}

The geometry of an aircraft's lifting surfaces defines its required power and hence its speed range during steady-level flight. To 

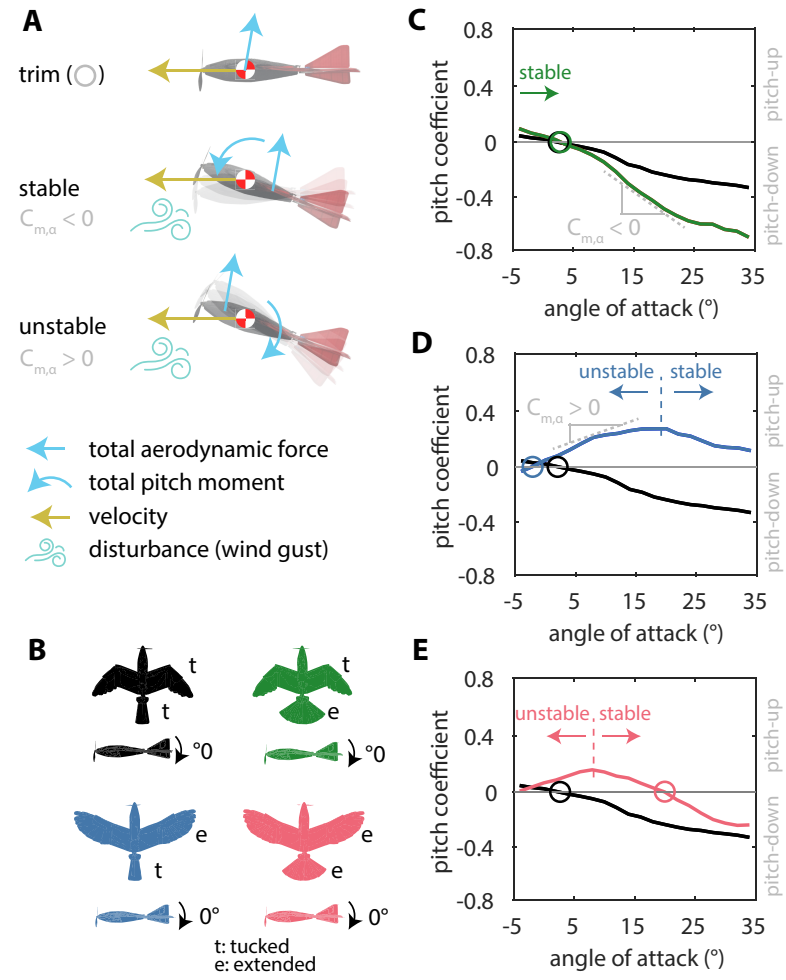

Fig. 5. Wing and tail morphing greatly changes pitch stability. Static wind tunnel data (flow velocity of $8.0 \mathrm{~m} / \mathrm{s}$ ). The gray lines in the plots indicate the zero moment. $C_{m, \alpha}$ indicates the pitch coefficient slope. (A) Trim flight is achieved when the pitch moment is zero. Pitch stability is achieved when there is an inherent tendency to return to trim flight after a perturbation (i.e., a wind gust). The figure was adapted from (24). (B) We tested four different configurations: tucked wing and tail (black), tucked wing and extended tail (green), extended wing and extended tail (blue), and extended wing and tail (red). The tail elevation was set to $0^{\circ}$. (C) Extending the tail decreases the pitch coefficient slope (green line) and shifts the trim point by $<0.5^{\circ}$ (green circle), as compared with the tucked wing and tail (black line and circle). (D) Extending the wing causes a positive pitch coefficient slope (unstable) at low angles of attack and a negative pitch coefficient slope at high angles of attack. (E) Extending the wing leads to a stable trim at $20^{\circ}$ angle of attack.

analyze the power requirement and the speed range of avian-inspired wing and tail morphing, we defined a model of the LisHawk drone based on our wind tunnel measurements (see Materials and Methods). Then, we performed three morphology optimization studies (Fig. 6A) in simulation that searched the space of morphological configurations (control inputs; Fig. 6, C to E), resulting in the lowest power requirement (minimized output, Fig. 6B) for different possible flight speeds (Materials and Methods).

As expected, the aircraft with the tucked wing and tail configuration requires notably less power for high-speed flight (Fig. 6B, black line) than the extended wing and tail configuration, resulting from its comparatively higher lift-to-drag ratio (Fig. 1F, black line). The optimal tail elevation angle is negative (i.e., the tail deflects upward) and further decreases for decreasing speeds (Fig. 6E, black line). Because of the tail's $-20^{\circ}$ elevation limit (Fig. $6 \mathrm{E}$; see constraints in Materials and Methods) and the negative lift produced by the negative tail angle of attack (Fig. 6H, black line), the drone cannot produce sufficient lift to fly at speeds below $7.6 \mathrm{~m} / \mathrm{s}$ (Fig. 6B).
A
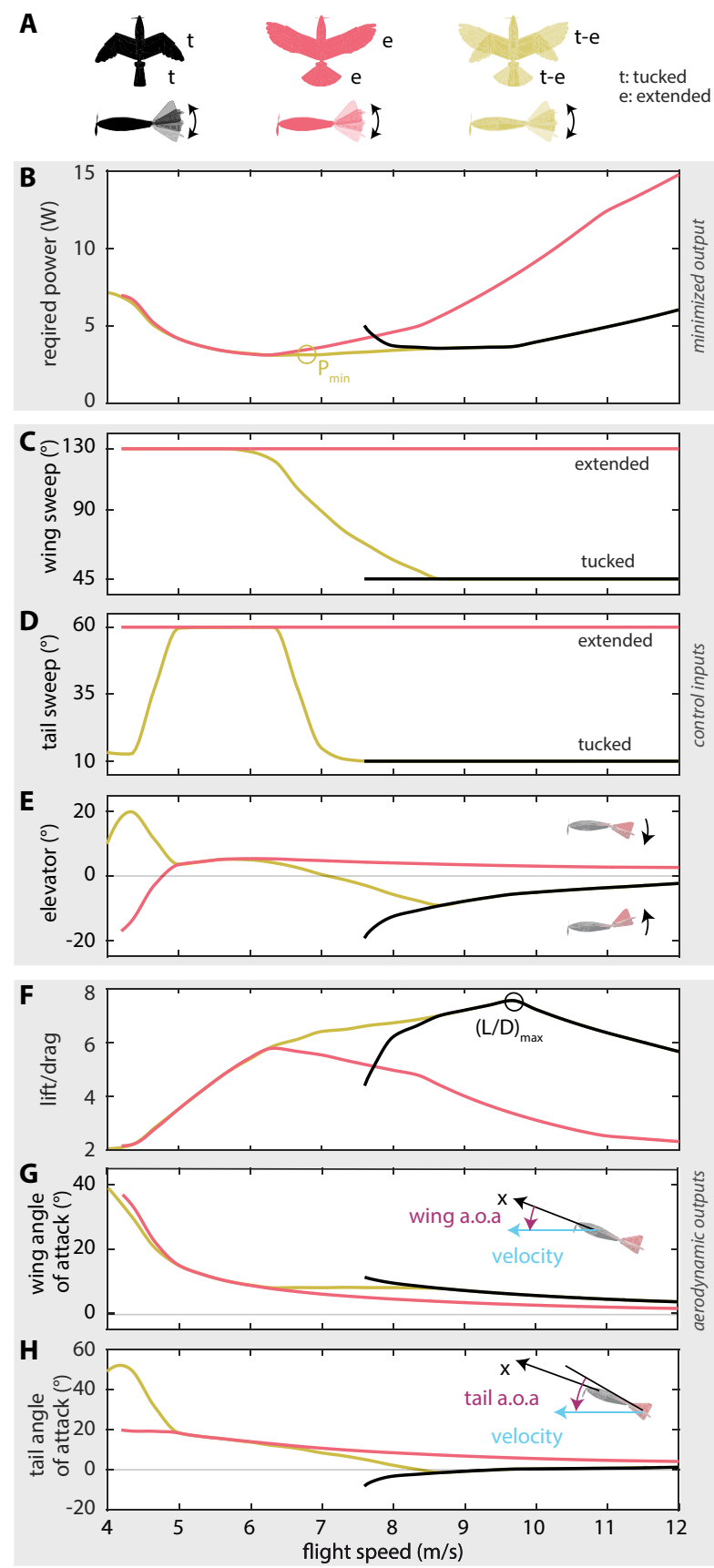

Fig. 6. The optimal wing and tail morphing reduces the required power and the minimum flight speed during steady-level flight. (A) We tested three different configurations: optimal tail elevation with fixed tucked wing and tail (black); optimal tail elevation with fixed extended wing and tail (red); and optimal tail elevation, wing morphing, and tail morphing (yellow). (B) Wing and tail morphing decreases the power requirement at high flight speeds. (C) Between 6.2 and $8.6 \mathrm{~m} / \mathrm{s}$, the wing area is increasing from tucked to extended to support the drone weight at lower flight speeds. (D) Below $7.6 \mathrm{~m} / \mathrm{s}$, the tail area increases to support the drone weight. (E) To achieve steady-level flight, with a tucked wing and tail, the elevator is always deflected upward, whereas with an extended wing and tail, the elevator is deflected downward. (F) The best lift-to-drag ratio is achieved with the tucked wing and tail at $9.8 \mathrm{~m} / \mathrm{s}$. (G) When approaching their minimum speed, all test configurations must increase the wing angle of attack to support the drone's weight. $(\mathbf{H})$ When the wing and tail are extended, the tail operates at positive angles of attack. 
The aircraft with the extended wing and tail can fly substantially slower (up to $4.2 \mathrm{~m} / \mathrm{s}$; Fig. $6 \mathrm{~B}$, red line) than the tucked wing and tail configuration because of its comparatively higher lift (Fig. 6F, red line). The optimal tail elevation angle is negative only for slow speeds (below $4.8 \mathrm{~m} / \mathrm{s}$ ) and is positive for increasing speeds (Fig. 6E, red line). Nevertheless, due to the high wing angles of attack at slow speeds (Fig. 6G), the tail angle of attack is positive over the entire speed range (Fig. $6 \mathrm{H}$ ). Therefore, when the wing and tail are extended, the tail always contributes to the overall lift.

The simultaneous optimization of tail elevation, wing sweep, and tail sweep (Fig. 6A, yellow) yields aircraft configurations that are equal to the tucked wing and tail above $8.6 \mathrm{~m} / \mathrm{s}$ and to the extended wing and tail between 5 and $6.2 \mathrm{~m} / \mathrm{s}$ (Fig. 6, B to H). Between flight speeds of 6.2 and $8.6 \mathrm{~m} / \mathrm{s}$, the optimal morphing of wing, tail, and elevation (Fig. 6, C to E, yellow line) can reduce the aerodynamic trade-offs between the extended and tucked configurations, thus considerably decreasing the power requirement (Fig. 6B, yellow line) by increasing the lift-to-drag ratio (Fig. $6 \mathrm{~F}$, yellow line). For speeds below $5.0 \mathrm{~m} / \mathrm{s}$, the tail is deflected further downward (Fig. 6E, yellow line) and its sweep angle is reduced (Fig. 6D, yellow line), resulting in a slightly lower power and slower speed $(4.0 \mathrm{~m} / \mathrm{s})$ than the extended wing and tail configuration (Fig. 6B, yellow line). However, we think that this result should be treated with caution. Aerodynamic measurements at high angles of attack are noisier (see SDs in figs. S4 and S5) because of the more turbulent flow conditions. Therefore, this small change in the power curve in combination with the difference in morphology may have resulted from noisier data. acceleration and speed in the drone's $x z$ plane, pitch acceleration and speed, and flight trajectory. Each maneuver was initiated from trim flight in the baseline condition of tucked wing and tail at about $10 \mathrm{~m} / \mathrm{s}$, and the drone was then morphed into one of the three wing and tail configurations shown in Fig. 7A.

Overall, the pull-up experiments confirm the wind tunnel results. In the control condition where both wing and tail are tucked, the upward deflection of the tail generates a small and gradual increment in linear and pitch accelerations (Fig. 7, C and E, black line), which respectively result in decreasing linear speed and increasing pitch speed (Fig. 7, D and F, black line). In this condition, the smallest pull-up radius measured during the maneuver is $14.3 \mathrm{~m}$ (Fig. 7B, black line). In the condition with tucked wing and extended tail, the larger tail surface produces a larger pitch-up moment (Fig. 7C, green line), which generates larger linear and pitch accelerations at the maneuver onset (Fig. 7, C and E, green line) that respectively result in faster decreasing linear speed and faster increasing pitch speed (Fig. 7, D and F, green line). In this condition, the smallest pull-up radius measured during the maneuver is reduced to $5.7 \mathrm{~m}$ (Fig. 7B, green line). However, we observed the largest linear and angular acceleration when the maneuver is performed with the extended wing and tail (Fig. 7, C and E, red line). In this configuration, the pitch-up moment is the largest (Fig. 4E, red line), which results in the fastest decreasing linear speed and fastest increasing pitch speed (Fig. 7, D and F, red line). In this condition, the smallest pull-up radius measured during the maneuver is only $1.0 \mathrm{~m}$ (Fig. 7B, red line).

\section{DISCUSSION}

Results obtained from wind tunnel experiments, morphology optimization studies, and flight tests indicate that avian-inspired

\section{Flight tests}

To assess the drone performance during aggressive flight as a function of different wing and tail configurations in real-world flights, we performed pull-up maneuvers (movie S4) and measured linear
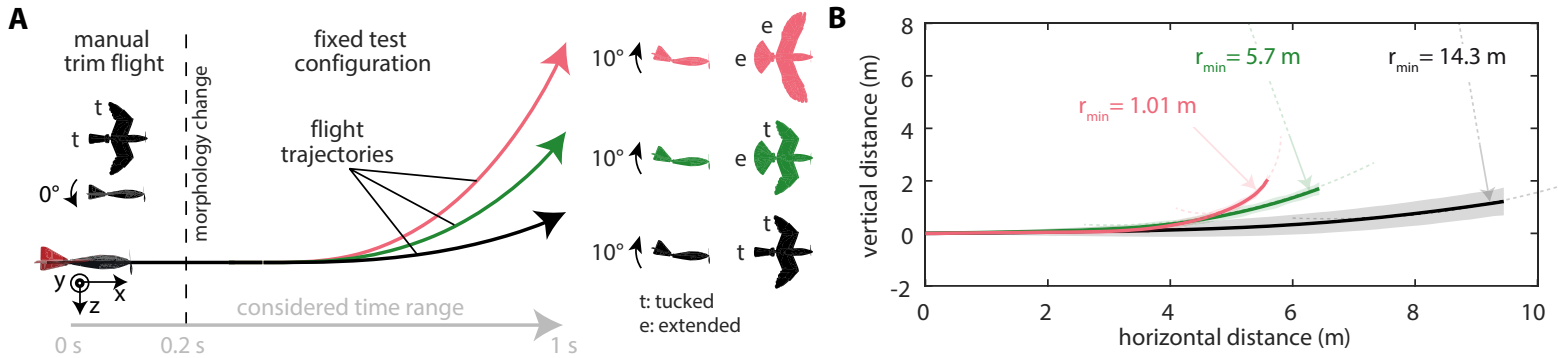

C
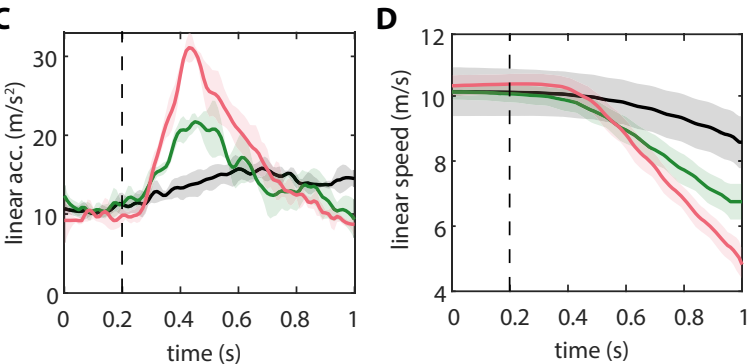

$\mathbf{F}$

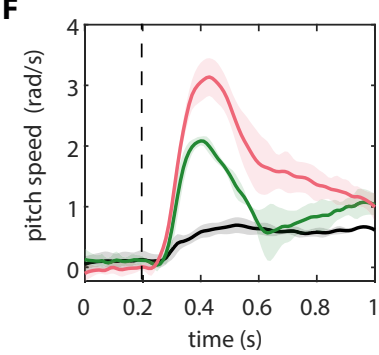

Fig. 7. Wing and tail morphing increases flight performance during pull-up maneuvers. The shaded areas indicate the SD from four trial runs. (A) Exemplary illustration of the pull-up maneuver. The maneuver was initiated from trim flight before changing into three different wing and tail configurations, while the tail was deflected upward by $10^{\circ}$ : tucked wing and tail (black), tucked wing and extended tail (green), and extended wing and tail (red). Each configuration was tested four times. (B) Flight trajectory flown for all three configurations and the minimum radius after $0.8 \mathrm{~s}$. (C) Initial linear acceleration in the $x z$ plane (and thus maneuverability) is increased by $270 \%$ when morphing from tucked to extended wing and tail. (D) The flight speed is greatly reduced when changing the wing and tail morphology. (E) The initial pitch acceleration (and thus pitch agility) is increased in magnitude by $445 \%$. (F) The increased pitch acceleration results in a notable increase in pitch speed. 
morphing of the drone's wing and tail can notably improve flight performance in two diverse flight regimes, namely, cruise and aggressive flight. In cruise flight, the required power should be small for highspeed area coverage and long-range flight, which is achieved by morphing from extended to tucked wing and tail above $7.8 \mathrm{~m} / \mathrm{s}$ (Fig. 6, C and D). For instance, for a 5-W thrust power requirement, the drone with the tucked wing and tail configuration can fly $31.4 \%$ faster than the drone with the extended wing and tail configuration (Fig. 6B). Furthermore, when flying at $9.6 \mathrm{~m} / \mathrm{s}$ [maximum range speed due to best lift-to-drag ratio (8)], the change from extended to tucked wing and tail configuration results in $55.4 \%$ less power requirement, which also indicates a notable range increase. This morphology also reduces the exposed wing area (Fig. 2C) and increases the pitch stability (Fig. 5C). Therefore, as previous literature has shown $(10,24)$, tucking the wing and tail may reduce the sensitivity to wind gusts.

In contrast, aggressive flight requires both high maneuverability and agility to perform sharp course variations. At high flight speeds, the tucked wing and tail configuration can produce sufficient aerodynamic forces and moments for aggressive flight because the aerodynamic forces and moments scale with the square of the flight speed (8), but at medium to low flight speeds, it cannot. To increase maneuverability and agility at low speed, the drone can unfold the wing and tail, which improves maneuverability by increasing the maximum lift up to $70.8 \%$ and the corresponding drag up to $63.5 \%$ (Fig. 3C). The extended wing and tail configuration also improves agility by increasing the pitch-up moment up to $169.7 \%$, which remarkably remains positive even at $34^{\circ}$ angle of attack (Fig. 4F). The change from tucked to extended wing and tail also creates a pitch instability below $8^{\circ}$ angle of attack (Fig. 5E), thus facilitating even greater pitch moments.

The ability of the avian-inspired drone's tail to produce lift is particularly noteworthy. In a conventional aircraft, the horizontal tail's role is limited to maintaining pitch stability and control. On the other hand, biological studies on the avian tail suggested that it contributes to the bird's overall lift $(14,49,22)$, thus improving its maneuverability and slow flight capabilities. Our study shows the lifting effects of the tail when operated in synergy with the wing on a drone. The results of our morphology optimization studies indicate that when the wing is extended, the LisHawk's tail operates at positive angles of attack, and even at increased angles of attack with respect to the main wing, depending on the flight speed (Fig. 6, $\mathrm{G}$ and $\mathrm{H}$ ). Therefore, the tail produces positive lift and contributes to the overall lift force (Fig. 3E). This synergistic lift production by the tail and the wing enables the avian-inspired drone to fly at a $44 \%$ lower speed than the tucked wing and tail configuration (Fig. 6B).

The extended maneuverability and agility produced by wing and tail morphing could also be useful in other maneuvers performed by birds, such as perching. Fixed-wing drones must rapidly pitch up to greatly increase drag and rapidly decelerate before perching $(31,50)$. However, it is challenging for fixed-wing drones to pitch up rapidly and to maintain controlled flight in those conditions during the final approach. Instead, the avian-inspired, morphing drone described here can rapidly pitch up, operate at high angles of attack, and achieve stable trim flight at higher angles of attack by extending wing and tail (for example, see the landing maneuver at the end of movie S5). This flight capability, also called supermaneuverability (51), could be applied to reduce the landing footprint of winged drones.
Flight tests confirmed a substantial increase in maneuverability and agility during a pull-up maneuver (Fig. 7, C and E; see movie S5 for additional flight maneuvers); however, manual teleoperation did not allow us to fully leverage the flight potentials of the drone. For example, we did not fly the drone with an extended wing and a tucked tail, even though this combination had been shown to be the most agile at high angles of attack (Fig. 5D, blue line). The drone became unstable below $20^{\circ}$ angle of attack and was thus too difficult for a human operator to control. Furthermore, although the upward deflecting tail increases agility by increasing the pitch-up moment (Fig. 4C, black and green line), it also decreases maneuverability because it produces a negative lift (Fig. 3E, light red line). This configuration requires optimal control of the tail deflection throughout the maneuver to minimize the trade-off between agility and maneuverability. We believe that the full flight potential of drones with wing and tail morphing could be leveraged by advanced control and machine learning methods that will generate autopilots capable of continuously controlling and optimally adapting the machine morphing surfaces in stable and unstable flight based on sensory feedback $(48,52)$. Last, we think that our avian-inspired, morphing drone made of scalable, feathered folding structures and a mechanically simple and reliable propulsion system paves the way for a generation of aircraft design that could adapt flight configuration to cover long distances, precisely land in small areas, and fly in confined spaces as birds do.

\section{MATERIALS AND METHODS Artificial feathers}

We manufactured artificial feathers that are composed of three major parts (Fig. 2B): First, we cut a skeleton from a $0.3-\mathrm{mm}$ fiber glass sheet with a $\mathrm{CO}_{2}$ laser cutter (Trotec Speedy 400). This material was chosen because it combines a low mass, flexibility, and sufficient stiffness to absorb the aerodynamic loads when the feathers are slightly overlapped. Second, we covered the skeleton with an airtight and tear-resistant membrane (ripstop polyester fabric) and fixed it with cyanoacrylate glue (viscosity, 3 to $10 \mathrm{mPa} \cdot \mathrm{s}$ ). Last, we fixed a $1.5-\mathrm{mm}$ carbon tube (wall thickness of $0.5 \mathrm{~mm}$ ) onto the skeleton to act as a shaft.

\section{Morphing wing}

Birds can maintain pitch stability (pitch moment curve with a negative slope) and balance (positive lift when in trim) during gliding flight solely through the geometry of their main wing $(14,24)$. This aerodynamic characteristic improves the versatility of the bird's horizontal tail (for example, to generate lift) compared with traditional aircraft, where the tail acts solely as a pitch stabilizer and elevator (21). Hence, we designed the LisHawk wing to be pitch stable and balanced. To do so, we used the XFLR5 software to perform preliminary flow simulations for selecting appropriate wing airfoils. XFLR5 simulates the potential flow around the airfoil by using horseshoe vortex elements and gives an estimate of the aerodynamic forces and moments while ignoring viscous effects. We considered the largest aspect ratio wing (symmetric wing sweep of $90^{\circ}$ ) as the design point (nearly median setting between tucked and extended wing), whereas the tail was not modeled. The wing had to be distinguished in three different sections (see fig. S1): (i) an outer section, composed of overlapping, artificial feathers, which is similar to a flat plate. (ii) A center wing section accommodating the wing morphing mechanism: It is 
composed of a thick frontal section accommodating this morphing mechanism and a feathered rear section. Mimicking the northern goshawk airfoil (43) would not have provided sufficient thickness to accommodate the morphing mechanism and would have induced a large negative pitching moment due to the large camber. To address this, we adapted a thicker eagle airfoil (53) and adjusted the camber to $1.5 \%$ to decrease the negative pitching moment at the cost of reduced aerodynamic performance (21). (iii) An inner wing section consisting of a rigid EPP body: Here, we wanted to apply an established low-Reynolds number airfoil, which complies with the stability and balance requirements set by the outer two wing sections. We chose the JWL-065, which is a flying wing airfoil.

The fabrication process of the morphing wing mechanism (Fig. 2G) is depicted in movie S1. For the hinge, we used a 3D-printed (Stratasys Dimension Elite) acrylonitrile butadiene styrene (ABS) part that is reinforced by two $0.5-\mathrm{mm}$ glass fiber plates (cut by $\mathrm{CO}_{2}$ laser cutter). For the wrist skeleton, we chose a balsa-glass fiber (material thickness: balsa, $6 \mathrm{~mm}$; glass fiber, $0.7 \mathrm{~mm}$ ) composite structure, which entails both low weight and high rigidity. To reinforce the wrist skeleton further, we wrapped the most stressed parts with Dyneema $(0.2 \mathrm{~mm}, 20 \mathrm{~kg})$. We then connected the bearings $(D=7 \mathrm{~mm}, d=3 \mathrm{~mm}$, $W=3 \mathrm{~mm}$ ) with the glass fiber plates of the wrist compound by using custom-made ABS rings, which were fixed with cyanoacrylate glue (viscosity, 3 to $10 \mathrm{mPa} \cdot \mathrm{s}$ ). The feathers were attached to feather pins, which, in return, were attached to the skeleton via 2-mm carbon shafts. Inviscid cyanoacrylate glue was used to interconnect parts.

\section{Morphing tail}

The fabrication process of the morphing and deflecting tail, shown in Fig. 2I, consists of the steps shown in movie S2. For the tail skeleton, we manufactured interlocking elements from a $0.5-\mathrm{mm}$ glass fiber (cut with the $\mathrm{CO}_{2}$ laser cutter), which were fixed with cyanoacrylate glue. In doing so, we could nearly halve the weight with respect to a previous, three-dimensional (3D)-printed version. We made the pulleys, the feather pins, and the universal joint from $3 \mathrm{D}$-printed ABS, whereas we chose 2 - $\mathrm{mm}$ carbon pipes for all the feather pins. A Dyneema cable $(0.2 \mathrm{~mm}, 20 \mathrm{~kg})$ was used to connect the outer feather pin levers over the skeleton pulleys via a fuselage beam to the servo at the front of the fuselage.

\section{Fuselage}

We attached the morphing wing (via t-connectors) and tail (via universal joint) to a $8 \mathrm{~mm} \times 8 \mathrm{~mm}$ (wall thickness, $0.5 \mathrm{~mm}$ ) pultruded carbon fiber square tube in the center of the fuselage (Fig. 2A). This square tube was surrounded by an EPP shell with a wall thickness of $6 \mathrm{~mm}$. The fuselage was composed of six independent parts, which were cut around wooden patterns with a hot wire and then glued together with UHU por. The surface was smoothed with sandpaper and a hot iron to reduce the skin friction drag. The finished fuselage hid the structural and electrical components in a soft shell. We mounted the motor on the LisHawk's EPP fuselage and did not link it directly to the square tube (Fig. 2A). This way, we could dampen vibrations from the motor and make the drone more impact resilient.

\section{Drone avionics}

For the propulsion system, we chose a S-1805-2250KV Scorpion brushless DC motor (Fig. 2A, blue) with a $7 \times 6$ GWS propeller (Fig. 2A, black; nominal stationary thrust is $262 \mathrm{~g}$ with a $2 \mathrm{~S} 650 \mathrm{~mA} \cdot \mathrm{h}$ battery) and a Turnigy Plush 10A electronic speed controller (Fig. 2A, yellow). To actuate the wrist flexing mechanism, we chose two KST X08 Plus servos $[m=9 \mathrm{~g}, T=3.8 \mathrm{~kg} \cdot \mathrm{cm}$ (torque) at $6 \mathrm{~V}]$, whereas for the tail deflections and tail spreading, we used three BMS-306 digital servos $(m=7.1 \mathrm{~g}$, $T=2.0 \mathrm{kgcm}$ at $6 \mathrm{~V}$ ). We used PixRacer (Fig. 2A, red) for data logging in combination with a Drotek M8Q GPS block with antenna (Fig. 2A, green). We piloted the LisHawk drone with a Turnigy X9R PRO 2.4-GHz radio controller and an Orange RX110 DSMX/DSM2 satellite receiver (Fig. 2A, orange).

\section{Wind tunnel measurements}

We performed wind tunnel studies on the open-jet wind tunnel (WindShape) at the HES-SO University of Applied Sciences and Art Western Switzerland in Geneva. We set the air stream to the expected mean velocity of $8 \mathrm{~m} / \mathrm{s}$, which corresponds to a Reynolds number of 91,837 for the LisHawk drone. We mounted the LisHawk drone in its center of gravity on a RUAG Aerospace 6 component sting balance (Strain-Gauge Balance 204-6A), which was attached to a robotic arm to accurately and autonomously position the drone in the wind tunnel at the respective angle of attack. Measurements were taken between $-4^{\circ}$ and $34^{\circ}$ angle of attack at $2^{\circ}$ steps, whereas the angle of attack range was limited by the robotic arm's allowable range. We measured the aerodynamic forces and moments of all possible combinations of three different symmetric wing sweep angles of $45^{\circ}$, $90^{\circ}$, and $130^{\circ}$; three different tail sweep angles of $10^{\circ}, 35^{\circ}$, and $60^{\circ}$; and five different horizontal tail deflection angles of $-20^{\circ},-10^{\circ}, 0,10^{\circ}$, and $20^{\circ}$. Furthermore, we also measured the effect of asymmetric sweeping for two wing asymmetries of $45^{\circ}$ and $85^{\circ}$ (Fig. $4 \mathrm{H}$ ). For the data logging, we used an HBM MX840B universal amplifier and the Catman V5.2.2 software for data acquisition. Before a measurement sequence was started, we zeroed the data logging device in calm air conditions. Then, we measured the aerodynamic forces and moments for $4 \mathrm{~s}$ at $300 \mathrm{~Hz}$ after a setting time of $12 \mathrm{~s}$.

To analyze the wind tunnel force and moment data, we used a MATLAB script, which calculated the mean and SD of the aerodynamic force and moment over the 4-s measurement period. In addition, these results were nondimensionalized by using ( 8 )

$$
\begin{gathered}
C_{L}=\frac{2 L}{\rho S V^{2}} \\
C_{D}=\frac{2 D}{\rho S V^{2}} \\
C_{m}=\frac{2 Q}{\rho S c V^{2}} \\
C_{l}=\frac{2 P}{\rho S b V^{2}}
\end{gathered}
$$

$L$ is the lift force, $D$ is the drag force, $Q$ is the pitching moment, $P$ is the roll moment, $V$ is the air speed, $S$ is the wing area, $\rho$ is the air density, $c$ is the mean aerodynamic chord, and $b$ is the wingspan. We used the tucked wing and tail configuration $\left(S=0.117 \mathrm{~m}^{2}\right.$, mean aerodynamic chord $=0.161 \mathrm{~m}$ ) as a baseline. The SDs of the lift, drag, and pitch moment data shown in Figs. 3, 4, and 5 are provided in figs. S4 and S5.

During the wind tunnel testing, we did not capture propeller slipstream effects, because our focus was to understand the aerodynamics of the morphing surfaces. Studies of the propeller slipstream on drone flight performance have previously been published, for example, in $(54,55)$. These results suggest an improvement in 
aerodynamic efficiency (lift-to-drag ratio is increased) and in control effectiveness of the tail due to the increase in dynamic pressure. Consequently, we can expect that the aerodynamic behavior with the propeller slipstream is better than the wind tunnel results obtained without propeller slipstream.

\section{Maneuverability metric}

Maneuverability is a concept of linear motion. We define it as the ability to induce high controlled linear accelerations, that is, to rapidly change the linear velocity and direction of the flying body's translational movement (4). In the wind-fixed frame, it can be defined as (see Supplementary Text for mathematical formulation and assumptions)

$$
\left(\begin{array}{c}
\ddot{x} \\
\ddot{y} \\
\ddot{z}
\end{array}\right)=\left(\begin{array}{c}
\frac{-D}{m}-g \sin \alpha \\
-\frac{L \sin \varphi}{m} \\
\frac{L \cos \varphi}{m}-g \cos \alpha
\end{array}\right)
$$

where $D=D_{\text {wing }}+D_{\text {tail }}$ is the combined drag force of wing (including fuselage) and tail (Fig. 1B), $L=L_{\text {wing }}+L_{\text {tail }}$ is the combined lift force of wing (including fuselage) and tail (Fig. 1B), $m$ is the mass, $g$ is the gravitational constant, $\alpha$ is the angle of attack, and $\varphi$ is the bank angle.

\section{Agility metric}

Agility is a concept based on rotational motion. We define it as the ability to produce a high controlled angular rate in roll $\dot{p}$, pitch $\dot{q}$, and yaw $\dot{r}$, act around the body's fixed axes (fig. S2). To estimate external factors influencing agility, we simplified the kinematic equation of motion (see Supplementary Text for mathematical formulation and assumptions) to pure rotation (56) so that

$$
\left(\begin{array}{c}
\dot{p}_{p} \\
\dot{q}_{p} \\
\dot{r}_{p}
\end{array}\right)=\left(\begin{array}{c}
\frac{P}{I_{x x}} \\
\frac{Q}{I_{y y}} \\
\frac{R}{I_{z z}}
\end{array}\right)
$$

where $P$ is the roll moment, $Q$ is the pitch moment, and $R$ is the yawing moment acting around the aircraft-fixed axes. The denominator consists of the moment of inertia $I_{x x}$ around $x, I_{y y}$ around $y$, and $I_{z z}$ around $z$.

\section{Pitch stability}

Mathematically, an aircraft is stable in pitch if the slope of the pitch coefficient $C_{m}$ with respect to the angle of attack (Fig. 5, C to E) (5) is negative such that

$$
C_{m, \alpha}=\frac{\partial C_{m}}{\partial \alpha}<0
$$

Thus, if this applies, the aircraft is stable because the angle of attack will passively converge toward a stable equilibrium at $C_{m}=0$ after a disturbance. Contrarily, if the pitch coefficient slope is positive, the angle of attack will diverge from equilibrium and the aircraft is unstable. The independent contribution to pitch stability of wing and tail is discussed in Supplementary Text. It is common practice to evaluate the pitch stability of an aircraft in the stick-fixed (zero elevation, zero rudder) configuration, as shown in Fig. 5B (8).

\section{Morphology optimization}

We wanted to identify the optimal wing sweep, tail sweep, and elevator deflection angles to minimize the power requirement. To do so, we solved an optimization problem in simulation using MATLAB's MultiStart algorithm with the fmincon solver (50). This algorithm selects the best control inputs (wing sweep angle $\Delta_{\mathrm{w}}$, tail sweep angle $\Delta_{\mathrm{t}}$, elevator deflection angle $\Delta_{\mathrm{e}}$ ), which lead to an overall minimum power requirement (minimized output) through multiple searches of the local minimum from an extensive range of randomly selected initial points within the bounds defined in Table 1. Each simulation is performed for a set of flight speeds between 4 and $12 \mathrm{~m} / \mathrm{s}$ at steps of $0.2 \mathrm{~m} / \mathrm{s}$. Three different studies were performed: (i) The tail deflection angle was optimized, while the wing and tail were tucked; (ii) the tail deflection angle was optimized, while the wing and tail were extended; (iii) tail deflection angle, wing sweep angle, and tail sweep angle were optimized.

The required power is defined as

$$
P=T V
$$

where $T$ is the thrust and $V$ is the flight speed (8). Because the optimization framework is run at given flight speeds, the only variable to be optimized is the thrust force $T$, which is the cost function that we minimized.

To run the optimization framework, we developed a three-degree of freedom nonlinear flight dynamics model to describe the longitudinal motion of the feathered drone (see equations below) by using the wind tunnel's aerodynamic data (see wind tunnel section) and without considering propeller slipstream effects. The state variables $V, \alpha, \gamma, q, \dot{x}$, and $\dot{z}$ correspond to the aircraft's velocity, angle of attack, flight path angle, pitch rate, horizontal displacement, and vertical displacement, respectively. $\rho$ refers to the air density, and $g$ indicates the gravitational acceleration. $m, c$, and $S$ respectively describe the mass, mean aerodynamic chord, and reference wing area of the aircraft. $C_{m}$ is the pitch coefficient about the aircraft's center of gravity, and $I_{y y}$ is the mass moment of inertia. The differential equations describing the longitudinal aircraft motion are

\begin{tabular}{l|cc}
\hline $\begin{array}{l}\text { Table 1. Optimization variable bounds. } \\
\text { Lariable }\end{array}$ & Lower bound & Upper bound \\
\hline$\dot{x}(\mathrm{~m} / \mathrm{s})$ & 0 & 20 \\
\hline$\dot{z}(\mathrm{~m} / \mathrm{s})$ & -10 & 10 \\
\hline$q\left({ }^{\circ} / \mathrm{s}\right)$ & -115 & 115 \\
\hline$\alpha\left(^{\circ}\right)$ & -4 & 38 \\
\hline$\gamma\left({ }^{\circ}\right)$ & -90 & 90 \\
\hline$T / W$ & 0 & 0.7 \\
\hline$\delta_{w}\left({ }^{\circ}\right)$ & 45 & 130 \\
\hline$\delta_{t}\left({ }^{\circ}\right)$ & 10 & 60 \\
\hline$\delta_{e}\left({ }^{\circ}\right)$ & -20 & 20 \\
& & \\
\hline
\end{tabular}




$$
\begin{gathered}
\dot{V}=\frac{T \cos \alpha-m g \sin \gamma-\frac{1}{2} \rho V^{2} S C_{D}\left(\alpha, \delta_{e}, \delta_{t}, \delta_{w}\right)}{m} \\
\dot{q}=\frac{\frac{1}{2} \rho V^{2} S c C_{m}\left(\alpha, \delta_{e}, \delta_{t}, \delta_{w}\right)}{I_{y y}} \\
\dot{\alpha}=q-\frac{T \sin \alpha-m g \cos \gamma+\frac{1}{2} \rho V^{2} S C_{L}\left(\alpha, \delta_{e}, \delta_{t}, \delta_{w}\right)}{m V} \\
\dot{\gamma}=\frac{T \sin \alpha-m g \cos \gamma+\frac{1}{2} \rho V^{2} S C_{L}\left(\alpha, \delta_{e}, \delta_{t}, \delta_{w}\right)}{m V} \\
\dot{x}=V \cos \gamma \\
\dot{z}=-V \sin \gamma
\end{gathered}
$$

To ensure steady straight-level flight control solutions, we specified zero-equality constraints on the aircraft's pitch moment, pitch rate, flight path angle, and flight acceleration so that

$$
\begin{gathered}
\frac{1}{2} \rho V^{2} S c C_{m}\left(\alpha, \delta_{e}, \delta_{t}, \delta_{w}\right)=0 \\
\frac{T \cos \alpha-m g \sin \gamma-\frac{1}{2} \rho V^{2} S C_{D}\left(\alpha, \delta_{e}, \delta_{t}, \delta_{w}\right)}{m}=0 \\
\frac{T \sin \alpha-m g \cos \gamma+\frac{1}{2} \rho V^{2} S C_{L}\left(\alpha, \delta_{e}, \delta_{t}, \delta_{w}\right)}{m V}=0 \\
\gamma=0 \\
q=0
\end{gathered}
$$

\section{Flight test setup}

We launched the LisHawk by arm throw, and flights with a duration between 4 and 8 min were performed before landing on the ground (movie S5). As continuous control inputs, throttle for thrust, elevator for pitch, rudder for yaw, and asymmetric wing sweep for roll were available. We defined switches on the remote control to change the symmetric sweep of the morphing tail and main wing symmetrically during the flight to tucked and extended for the tail, and tucked, perpendicular, and extended for the wing (Fig. 2A).

For the pull-up study, we initiated the maneuver from trim with the tucked wing and tail and at a $60 \%$ thrust setting. Through a switch on the remote control, the pilot initiated the upward tail deflection $\left(-10^{\circ}\right)$ and the simultaneous adaption to the tucked wing and tail, tucked wing and extended tail, and extended wing and tail configuration. Through the PixRacer, we logged the linear acceleration, the rotational velocity, the control inputs, and the position [sampling rate inertial measurement unit (IMU), $100 \mathrm{~Hz}$; sampling rate GPS, $10 \mathrm{~Hz}$; maximum logging rate, $100 \mathrm{~Hz}$. We then used a MATLAB script to calculate linear speed ( $x z$ plane), pitch speed, and the mean values over time with their SDs. We aligned the horizontal and vertical flight trace when the elevator deflection was engaged and rotated each trial run to fly from left to right, as shown in Fig. 7B. We also aligned the linear acceleration, rotational acceleration, linear speed, and rotational speed when the elevator deflection was engaged
(Fig. 7, C to F). Because of GPS tracking errors, we had to exclude one trial from each run (five were done for each configuration). No autopilot or other autonomous flight-enhancing measures were implemented during flight. All flights took place on a large, open field in calm wind conditions.

\section{SUPPLEMENTARY MATERIALS}

robotics.sciencemag.org/cgi/content/full/5/47/eabc2897/DC1

Text

Fig. S1. Airfoil considerations.

Fig. S2. Coordinate systems and variables.

Fig. S3. Wing and tail contribution to pitch stability.

Fig. S4. SD of lift and drag plots.

Fig. S5. SD of pitch and roll plots.

Table S1. Full-stroke morphing times.

Movie S1. Morphing wing fabrication.

Movie S2. Morphing tail fabrication.

Movie S3. Morphing control surfaces.

Movie S4. Pull-up maneuver.

Movie S5. Flight test.

\section{REFERENCES AND NOTES}

1. D. Floreano, R. J. Wood, Science, technology and the future of small autonomous drones. Nature 521, 460-466 (2015).

2. J. Villasenor, "Drones" and the future of domestic aviation [Point of View]. Proc. IEEE 102, 235-238 (2014).

3. V. Kumar, N. Michael, Opportunities and challenges with autonomous micro aerial vehicles. Int. J. Robot. Res. 31, 1279-1291 (2012).

4. R. Dudley, Mechanisms and implications of animal flight maneuverability. Integr. Comp. Biol. 42, 135-140 (2002)

5. R. Brockhaus, W. Alles, R. Luckner, Flugregelung (Springer, ed. 3, 2011).

6. J. Verbeke, J. De Schutter, Experimental maneuverability and agility quantification for rotary unmanned aerial vehicle. Int. J. Micro Air Vehicles 10, 3-11 (2018).

7. A. A. Paranjape, N. Ananthkrishnan, Combat aircraft agility metrics-a review. J. Aerosp. Sci. Technol. 58, 143-154 (2006).

8. W. F. Phillips, Mechanics of Flight (John Wiley \& Sons, ed. 2, 2009).

9. B. N. Pamadi, Performance, Stability, Dynamics, and Control of Airplanes, Second Edition (American Institute of Aeronautics and Astronautics, 2004).

10. M. Di Luca, S. Mintchev, G. Heitz, F. Noca, D. Floreano, Bioinspired morphing wings for extended flight envelope and roll control of small drones. Interface Focus 7, 20160092 (2017).

11. M. Di Luca, S. Mintchev, Y. Su, E. Shaw, K. Breuer, A bioinspired Separated Flow wing provides turbulence resilience and aerodynamic efficiency for miniature drones. Sci. Robot. 5, eaay8533 (2020)

12. M. Abdulrahim, R. Lind, Modeling and control of micro air vehicles with biologicallyinspired morphing, in 2006 American Control Conference (IEEE, 2006).

13. S. Mintchev, D. Floreano, Adaptive morphology: A design principle for multimodal and multifunctional robots. IEEE Robot. Autom. Mag. 23, 42-54 (2016).

14. A. L. R. Thomas, G. K. Taylor, Animal flight dynamics I. Stability in gliding flight. J. Theor. Biol. 212, 399-424 (2001).

15. H. Hertel, Structure, Form, Movement (Reinhold Publishing Co., ed. 1, 1966).

16. A. L. R. Thomas, The flight of birds that have wings and a tail: Variable geometry expands the envelope of flight performance. J. Theor. Biol. 183, 237-245 (1996).

17. A. L. R. Thomas, The aerodynamic costs of asymmetry in the wings and tail of birds: Asymmetric birds can't fly round tight corners. Proc. R. Soc. Lond. B Biol. Sci. 254, 181-189 (1993).

18. U. M. Norberg, Vertebrate Flight: Mechanics, Physiology, Morphology, Ecology and Evolution (Springer, 2012).

19. A. L. R. Thomas, On the aerodynamics of birds' tails. Philos. Trans. R. Soc. Lond. B Biol. Sci. 340, 361-380 (1993)

20. V. A. Tucker, Pitching equilibrium, wing span and tail span in a gliding Harris' hawk, Parabuteo Unicinctus. J. Exp. Biol. 165, 21-41 (1992).

21. K. Nickel, M. Wohlfahrt, Schwanzlose Flugzeuge: Ihre Auslegung und ihre Eigenschaften (Springer, 1990).

22. W. J. Maybury, J. M. V. Rayner, L. B. Couldrick, Lift generation by the avian tail. Proc. R. Soc. Lond. B Biol. Sci. 268, 1443-1448 (2001).

23. C. Berg, J. Rayner, The moment of inertia of bird wings and the inertial power requirement for flapping flight. J. Exp. Biol. 198, 1655-1664 (1995).

24. C. Harvey, V. B. Baliga, P. Lavoie, D. L. Altshuler, Wing morphing allows gulls to modulate static pitch stability during gliding. J. R. Soc. Interface 16, 20180641 (2019). 
25. S. M. Gatesy, K. P. Dial, From frond to fan: Archaeopteryx and the evolution of short-tailed birds. Evolution 50, 2037-2048 (1996).

26. C. J. Pennycuick, A wind-tunnel study of gliding flight in the pigeon Columba Livia. J. Exp. Biol. 49, 509-526 (1968).

27. D. Lentink, U. K. Müller, E. J. Stamhuis, R. de Kat, W. van Gestel, L. L. M. Veldhuis, P. Henningsson, A. Hedenström, J. J. Videler, J. L. van Leeuwen, How swifts control their glide performance with morphing wings. Nature 446, 1082-1085 (2007).

28. V. A. Tucker, Gliding flight: Speed and acceleration of ideal falcons during diving and pull out. J. Exp. Biol. 201, 403-414 (1998).

29. S. Barbarino, O. Bilgen, R. M. Ajaj, M. I. Friswell, D. J. Inman, A review of morphing aircraft. J. Intell. Mater. Syst. Struct. 22, 823-877 (2011).

30. A. S. Saeed, A. B. Younes, C. Cai, G. Cai, A survey of hybrid unmanned aerial vehicles. Prog. Aerosp. Sci. 98, 91-105 (2018).

31. C. Greatwood, A. Waldock, T. Richardson, Perched landing manoeuvres with a variable sweep wing UAV. Aerosp. Sci. Technol. 71, 510-520 (2017).

32. Z. R. Manchester, J. I. Lipton, R. J. Wood, S. Kuindersma, A variable forward-sweep wing design for enhanced perching in micro aerial vehicles, in 55th AIAA Aerospace Sciences Meeting (AIAA, 2017).

33. R. Siddall, A. Ortega Ancel, M. Kovač, Wind and water tunnel testing of a morphing aquatic micro air vehicle. Interface Focus 7, 20160085 (2017)

34. E. Chang, L. Y. Matloff, A. K. Stowers, D. Lentink, Soft biohybrid morphing wings with feathers underactuated by wrist and finger motion. Sci. Robot. 5, eaay1246 (2020).

35. A. Paranjape, J. Kim, N. Gandhi, S.-J. Chung, Experimental demonstration of perching by an articulated wing MAV, in AIAA Guidance, Navigation, and Control Conference (American Institute of Aeronautics and Astronautics, 2011).

36. A. A. Paranjape, S.-J. Chung, H. H. Hilton, A. Chakravarthy, Dynamics and performance of tailless micro aerial vehicle with flexible articulated wings. AIAA J. 50, 1177-1188 (2012).

37. M. Abdulrahim, H. Garcia, R. Lind, Flight characteristics of shaping the membrane wing of a micro air vehicle. J. Aircr. 42, 131-137 (2005).

38. H. Garcia, M. Abdulrahim, R. Lind, Roll control for a micro air vehicle using active wing morphing, in AIAA Guidance, Navigation, and Control Conference and Exhibit (American Institute of Aeronautics and Astronautics, 2003).

39. D. Li, S. Zhao, A. Da Ronch, J. Xiang, J. Drofelnik, Y. Li, L. Zhang, Y. Wu, M. Kintscher, H. P. Monner, A. Rudenko, S. Guo, W. Yin, J. Kirn, S. Storm, R. De Breuker, A review of modelling and analysis of morphing wings. Prog. Aerosp. Sci. 100, 46-62 (2018).

40. K. Herzog, Anatomie und Flugbiologie der Vogel (VEB Gustav Fischer Verlag, 1968).

41. R. Kenward, The Goshawk (T. and A. D. Poyser, 2010).

42. P. L. Kennedy, Northern Goshawk (Accipiter gentilis atricapillus): A Technical Conservation Assessment (USDA Forest Service, 2003).

43. J. J. Videler, Avian Flight (Oxford Univ. Press, 2005).

44. G. Cai, J. Dias, L. Seneviratne, A survey of small-scale unmanned aerial vehicles: Recent advances and future development trends. Unmanned Syst. 2, 175-199 (2014).

45. G. Sachs, Comparison of power requirements: Flapping vs. fixed wing vehicles. Aerospace 3, 31 (2016).

46. W. Shyy, Y. Lian, J. Tang, D. Viieru, H. Liu, Aerodynamics of Low Reynolds Number Flyers (Cambridge Univ. Press, 2008).
47. J. Rom, High Angle of Attack Aerodynamics-Subsonic, Transonic and Supersonic Flows (Springer, ed. 1, 1992)

48. D. E. Alexander, Nature's Flyers: Birds, Insects and the Biomechanics of Flight (The John Hopkins University Press, 2002).

49. J. R. Usherwood, J. A. Cheney, J. Song, S. P. Windsor, J. P. J. Stevenson, U. Dierksheide A. Nila, R. J. Bomphrey, High aerodynamic lift from the tail reduces drag in gliding raptors. J. Exp. Biol. 223, jeb214809 (2020).

50. M. Feroskhan, T. H. Go, Dynamics of sideslip perching maneuver under dynamic stall influence. Aerosp. Sci. Technol. 50, 220-233 (2016).

51. M. J. Abzug, E. E. Larrabee, Airplane Stability and Control: A History of the Technologies That Made Aviation Possible (Cambridge Univ. Press, ed. 2, 2002).

52. A. Waldock, C. Greatwood, F. Salama, T. Richardson, Learning to perform a perched landing on the ground using deep reinforcement learning. J. Intell. Robot. Syst. 92, 685-704 (2018).

53. A. C. Carruthers, S. M. Walker, A. L. R. Thomas, G. K. Taylor, Aerodynamics of aerofoi sections measured on a free-flying bird. Proc. Inst. Mech. Eng. G J. Aerosp. Eng. 224, 855-864 (2010).

54. J. M. Levin, A. A. Paranjape, M. Nahon, Sideslip and slipstream in extreme maneuvering with fixed-wing unmanned aerial vehicles. J. Guid. Control Dyn. 41, 1610-1616 (2018).

55. T. Bouquet, R. Vos, Modeling the propeller slipstream effect on lift and pitching moment, in 55th AlAA Aerospace Sciences Meeting (AIAA, 2017).

56. R. C. Nelson, Flight Stability and Automatic Control (McGraw Hill, ed. 2, 1998).

57. J. R. Squires, R. T. Reynolds, Northern Goshawk (Accipiter gentilis) (The Cornell Lab of Ornithology, 1997).

58. T. Edelsten, Northern goshawk in flight with extended wing and tail; www.birdskorea. org/Birds/Birdnews/BK-BN-birdnews-2009-10.shtml [accessed 2 February 2020].

59. R. Mangersnes, Juvenile northern goshawk (accipier gentilis) landing on a branch in reed bed; www.naturepl.com [accessed 2 October 2020].

Acknowledgments: We thank G. Catry, N. Bosson, S. Marquez, and A. Gros from WindShape for their continuous support when using their wind tunnel facility. We would also like to thank V. Ramachandran for his help during the writing process. Funding: This work was funded by the Swiss National Science Foundation through the National Center of Competence in Research Robotics. Author contributions: E.A., M.F., S.M., and D.F. developed the concept and wrote the manuscript. E.A. designed and fabricated the LisHawk drone. E.A., M.F., and F.N. designed the experiment and performed experimental wind tunnel tests. M.F. developed the test environment for the shape optimization study and performed simulations. E.A. conducted and analyzed the flight tests. Competing interests: The authors declare that they have no competing interests. Data and materials availability: All data needed to evaluate the conclusions of the paper are available in the paper or in the Supplementary Material.

Submitted 16 April 2020

Accepted 28 September 2020

Published 28 October 2020

$10.1126 /$ scirobotics.abc2897

Citation: E. Ajanic, M. Feroskhan, S. Mintchev, F. Noca, D. Floreano, Bioinspired wing and tail morphing extends drone flight capabilities. Sci Robot. 5, eabc2897 (2020). 